\title{
Mixed cultures of allogeneic dendritic cells are phenotypically and functionally stable - a potential for primary cell-based "off the shelf" product generation
}

\author{
URBAN ŠVAJGER ${ }^{I, 2}$, PRIMOŽ J. ROŽMAN ${ }^{I}$ \\ ${ }^{1}$ Blood Transfusion Center of Slovenia, Ljubljana, Slovenia \\ ${ }^{2}$ Faculty of Pharmacy, University of Ljubljana, Ljubljana, Slovenia
}

\begin{abstract}
Vaccination against tumors using antigen-pulsed dendritic cell $(D C)$ vaccines has greatly evolved over the last decade, with hundreds of active human clinical trials well on the way. The use of an autologous source for DC-based vaccine therapeutics remains the obvious choice in the majority of clinical studies; however, novel evidence suggests that an allogeneic source of DCs can yield success if administered in the right context. One of the challenges facing successful DC vaccination protocols is the generation of large enough numbers of DCs intended for vaccination and standardization of these procedures. In addition, variations in the quality of DC vaccines due to donor-to-donor variation represent an important therapeutic factor. To this day it has not been shown whether DCs from different donors can readily co-exist within the same co-culture for the extended periods required for vaccine manufacture. We demonstrate that generation of allogeneic DC co-cultures, generated from multiple unrelated donors, allows the preservation of their phenotypical and functional properties in vitro for up to 72 hours. Therefore, in the case of an allogeneic vaccination approach, one could ensure large numbers of DCs generated from a primary cell source intended for multiple vaccinations. By generating large amounts of ex vivo manufactured DCs from multiple donors, this would represent the possibility to ensure sufficient amounts of equipotent "off the shelf" product that could e.g. be used for an entire cohort of patients within a study.
\end{abstract}

Key words: dendritic cells, allogeneic, cancer vaccines, tumor immunity.

(Cent Eur J Immunol 2021; 46 (2): 152-161)

\section{Introduction}

Due to their unique antigen (Ag)-presenting capability, dendritic cells (DCs) as professional antigen-presenting cells (APCs) have made their way towards being one of the most promising tools for anti-tumor vaccination. Today, DCs are used in a great number of studies in vaccination protocols for the treatment of cancer [1]. The use of monocyte-derived DCs (MoDCs) still remains the most popular platform for DC vaccine manufacture; however, the source of monocytes as their progenitors, can differ with respect to whether they are autologous, i.e. originate from the patient himself, or allogeneic, where they can be obtained from appropriate related or unrelated donors.

There is still no clear consensus on which type of DC vaccine (either autologous - autoDCs or allogeneic - alloDCs) is more suitable for vaccination and there are no comparative studies describing the advantages or disad- vantages of using autoDCs vs. alloDCs, thereby showing the efficiency of one over the other. Although most clinical trials so far have focused on the use of autoDCs, various results in recent years highlighted some potential benefits of applying alloDCs, which should not be overlooked. In this manner, results from past clinical studies have shown that administration of allogeneic MoDC-based anti-cancer vaccines is feasible, safe and well tolerated [2-5]. For instance, an important advantage of using alloDCs is the feasibility of preparing large quantities of cells in comparison to autoDCs, where the patient, frequently exhausted from disease, cytotoxic drugs and invasive medical interventions, is the only source of biologic material. Indeed, DC dosage in a single vaccine can be substantial, in many cases ranging from 10 to 100 million DCs (source: National Institutes of Health [NIH]), depending on the route of administration. In addition, most protocols are designed

Correspondence: Prof. Urban Švajger, Blood Transfusion Center of Slovenia, Šlajmerjeva 6, 1000 Ljubljana, Slovenia,

e-mail: urban.svajger@ztm.si

Submitted: 30.06.2020; Accepted: 12.01.2021 
to offer multiple periodical vaccinations. It should also be noted that the number of DCs needed for vaccination is associated with more than twice the number of monocytes needed for their generation $[6,7]$. As good manufacturing practice (GMP)-compliant immunomagnetic monocyte isolation, which grants elevated numbers of highly pure DC precursors, is financially demanding, classic approaches, particularly isolation of monocytes by plastic adherence, are frequently used. This however leads to lower cell yields and sometimes difficulties in obtaining sufficient cell numbers for the entire vaccination protocol. Clearly, considering human leukocyte antigen (HLA) disparity between patients and an allogeneic DC vaccine, the use of multiple donors for DC generation would increase the chance of at least partial HLA compatibility.

In this report we demonstrate that upon optimal in vitro differentiation, MoDCs from multiple allogeneic donors can readily co-exist in co-culture for longer periods and exert similar phenotypic and functional features as their single-donor DC culture counterparts. These findings further facilitate the generation of large clinical-grade "off the shelf" batches that may be used for patients involved in a particular vaccination study, thereby alleviating and accelerating the standardization of such protocols, as well as lowering donor-to-donor variability.

\section{Material and methods}

\section{Cell culture and isolation}

Buffy coats from venous blood of normal healthy volunteers were obtained by the Blood Transfusion Centre of Slovenia, according to institutional guidelines. The study was approved by the National Medical Ethics Committee of the Ministry of Health, Republic of Slovenia, and written consent was obtained before each blood specimen collection.

Peripheral blood mononuclear cells (PBMCs) were isolated using Lympholyte-H (Cedarlane laboratories, Ontario, Canada). The cells were washed multiple times with Dulbecco's phosphate-buffered saline (DPBS), counted, and used as a source for isolation of $\mathrm{CD} 14^{+}$cells by adherence. Briefly, approx. 1/5 of the PBMCs isolated from one buffy coat were incubated for 2 hours at $37^{\circ} \mathrm{C}, 5 \% \mathrm{CO}_{2}$ in a $75 \mathrm{~cm}^{2}$ culture flask. Before the adherence step, the cells were washed several times with DPBS in order to eliminate most of the platelets, which can interfere with the process. After the adherence step, non-adherent cells were discarded and the flask was washed twice with DPBS at room temperature. The residual adherent cells (monocytes) were then cultured in X-VIVO15 culture medium (BiWhittaker, Lonza, USA) supplemented with $1 \%$ heat-inactivated human AB serum (prepared at the Blood Transfusion Centre of Slovenia, according to institutional guidelines). To differentiate monocytes from DCs, $800 \mathrm{U} / \mathrm{ml}$ of rhGM-CSF and $1000 \mathrm{U} / \mathrm{ml}$ of rhIL-4 (both Peprotech EC, London,
UK) were added. On day 2, half of the medium was exchanged with starting quantities of rhGMCSF $(800 \mathrm{U} / \mathrm{ml})$ and rhIL-4 (1000 U/ml). After 5 days, non-adherent, immature DCs (iDCs) were harvested and characterized by flow cytometry as CD1 $\mathrm{a}^{\text {low }}, \mathrm{CD} 14^{-}$, dendritic cell-specific intercellular adhesion molecule-3-grabbing non-integrin $(\mathrm{DC}-\mathrm{SIGN})^{+}, \mathrm{CD}^{-} 3^{-}, \mathrm{CD} 86^{\text {low }}$ and HLA-DR ${ }^{\text {low }}$. To activate iDCs, the cells were counted and re-suspended in X-VIVO15 containing $800 \mathrm{U} / \mathrm{ml}$ of rhGM-CSF and $20 \mu \mathrm{g} / \mathrm{ml}$ polyinosinic-polycytidylic acid (poly I:C) (Invivogen, San Diego, CA) and cultured for a further two days. In all experiments, monocytes were isolated and differentiated from four unrelated healthy donors simultaneously and then iDCs were prepared individually. Mixed allogeneic DC cultures were only set up upon complete monocyte to DC differentiation. To study mixed allogeneic DC cultures in comparison with each single DC culture, the cells were either left in their immature state with only granulocyte, macrophage colony-stimulating factor (GM-CSF) added, or activated with poly I:C for up to 72 hours, depending on the experiment.

Whole $\mathrm{CD}^{+} \mathrm{T}$ cells were isolated from each individual's PBMCs by immunomagnetic selection using CD4 microbeads (Miltenyi Biotec GmbH, Bergisch Gladbach, Germany), as described previously [8]. The purity of isolated $\mathrm{CD}^{+}$cells was always $>95 \%$, as determined by flow cytometry.

\section{Phenotypic characterization of DCs}

The levels of membrane expressed molecular markers were determined by flow cytometry using fluorescence labelled antibodies. On day 7 (two days after DC differentiation, 48 hours following single or mixed DC cultivation) single and mixed DC cultures containing either immature iDCs or poly I:C-activated mature DCs (mDCs) were harvested and cells collected by centrifugation. Appropriate antibodies (see below) were added to the cells, followed by incubation at room temperature for $15 \mathrm{~min}$ in the dark. The cells were then washed twice with DPBS and re-suspended in $2 \%$ paraformaldehyde. The following monoclonal antibodies were used: FITC labelled anti-CD1a, antiCD14, anti-CD80, and R-PE labelled anti-DC-SIGN (all from Biolegend, CA, USA), FITC labelled anti-CD83 (IQ Products, NL), FITC labelled anti-CD86 (DakoCytomation, Denmark), and R-PE labelled anti-HLA-DR (Exalpha Biologicals Inc., MA, USA). FITC-IgG1 and R-PE-IgG2a cocktail was used for isotype control (Biolegend). Results were expressed as mean fluorescence intensity (MFI) values after subtraction of the MFI value obtained with the control antibody.

\section{Apoptosis and cell death studies}

We validated the stability of mixed DC co-cultures in terms of potential changes in the extent of apoptotic and dead cells in comparison with single DC cultures. For this 
purpose DCs obtained from individual donors were cultured individually and jointly as a co-culture, for a period of 72 hours. Then the extent of apoptotic and dead DCs in each culture was determined by Annexin-V FITC conjugate and propidium iodide (Apoptosis assay kit, Invitrogen, Carlsbad, CA) staining and subsequent flow cytometry, using the FACSCalibur system (Becton Dickinson, Inc.).

\section{Morphological analysis}

Inverted light optical microscopy (Nikon Eclipse TE300, Tokyo, Japan) was performed in parallel to all other analyses to assess cell morphologies. Characteristic morphological features of in vitro monocyte-derived DCs were observed such as their characteristic shape and dendrite formation.

\section{Allogeneic $\mathbf{T}$ cell proliferation}

Either single or mixed donor DC cultures were used as in vitro stimulators of purified allogeneic $\mathrm{CD}^{+} \mathrm{T}$ cells as responders. DCs were either used in their immature state or activated for 48 hours with $20 \mu \mathrm{g} / \mathrm{ml}$ of poly I:C. P All assays, i.e. mixed lymphocyte cultures (MLCs), were performed in 96-well flat-bottom plates with a total volume of $200 \mu \mathrm{l} /$ well. In each well we seeded $1 \times 10^{4} \mathrm{DCs}$, after they had been washed twice with DPBS, and then added $1 \times 10^{5}$ allogeneic T cells. After 4 days of incubation $\left(37^{\circ} \mathrm{C} 5 \% \mathrm{CO}_{2}\right)$, the wells were pulsed with $1 \mu \mathrm{Ci} /$ well of ${ }^{3} \mathrm{H}$-thymidine (Perkin Elmer, Boston MA, USA) and $\mathrm{T}$ cell proliferation was measured following an additional 18-20 hours of incubation by liquid scintillation counting (Wallac microbeta counter, Turku, Finland).

\section{Activated $\mathbf{T}$ cell intracellular cytokine analysis}

To determine cytokine production by alloactivated responding $\mathrm{CD}^{+} \mathrm{T}$ cells on a single cell level we stained the cells intracellularly, using FITC-anti-IFN- $\gamma$, PE-anti-IL-10, PE-anti-IL-4 and PE-anti-IL-2 Abs (all from Invitrogen, Carlsbad, CA). For gating purposes we used Cy5-PE-antiCD4 Ab (Biolegend, CA, USA). Briefly, $1 \times 10^{5}$ DCs (either immature or mature, from single and mixed DC cultures) were co-cultured with $1 \times 10^{6}$ naïve $\mathrm{CD} 4{ }^{+} \mathrm{CD} 45 \mathrm{RA}^{+}$ $\mathrm{T}$ cells in 24-well tissue culture plates in complete medium (X-VIVO15 supplemented with $1 \%$ human AB serum). After 7 days, $\mathrm{CD} 4^{+} \mathrm{T}$ cells were collected and restimulated with $50 \mathrm{ng} / \mathrm{ml}$ phorbol myristate acetate (PMA) and $500 \mathrm{ng} / \mathrm{ml}$ ionomycin (both from Sigma). After 4 hours $10 \mu \mathrm{g} / \mathrm{ml}$ of Brefeldin A (Biolegend) were added for an additional 4 hours. Afterwards the cells were fixed with $4 \%$ PHA for 1 hour and then permeabilized using $0.1 \%$ Triton $\mathrm{X}-100$ for 10 minutes. Subsequently the cells were washed twice with DPBS. To prevent non-specific staining, T cells were incubated for $45 \mathrm{~min}$ in DPBS containing 3\% bovine serum albumin (BSA) and then stained with FITC-antiIFN- $\gamma$, PE-anti-IL-10, PE-anti-IL-4 and PE-anti-IL-2 Abs for $45 \mathrm{~min}$ in the dark. Before they were analyzed with the FACSCalibur flow cytometer system (Becton Dickinson, Inc.), the cells were washed twice with DPBS.

\section{Measurement of DC cytokine production}

Dendritic cells were differentiated and set up in either single or mixed DC cultures. Then they were either left in their immature state or further activated with $20 \mu \mathrm{g} / \mathrm{ml}$ of poly I:C, for 48 hours. Afterwards, cell-free culture supernatants were carefully collected and the levels of IL-10 and IL-12p70 measured by enzyme-linked immunosorbent assay (ELISA). The levels of both IL-10 and IL-12p70 were determined using Max Deluxe ELISA sets for IL-10 and IL-12p70 (Biolegend, San Diego, CA). All assays were set up in duplicates and performed in accordance with the manufacturer's protocols. The lower limit of IL-12p70 detection was $4 \mathrm{pg} / \mathrm{ml}$ and $3 \mathrm{pg} / \mathrm{ml}$ for IL-10, respectively.

\section{Results}

\section{Allogeneic DC co-cultures are stable and are not characterized by increased cell death}

Firstly, we wanted to determine the stability of co-cultures consisting of fully differentiated DCs from four allogeneic donors. After confirming optimal differentiation of monocytes to iDCs, by assessing the expression of CD1a $\mathrm{a}^{++}$, $\mathrm{CD}^{-} 4^{-}$and DC-SIGN ${ }^{++}$molecules (data not shown), the iDCs were further cultured for 72 hours, either as single donor or mixed donor cultures. These were either left untreated (except for the addition of GM-CSF) or stimulated, i.e. matured with $20 \mu \mathrm{g} / \mathrm{ml}$ of poly I:C. After 3 days, all cell cultures were collected and DCs stained for early/late apoptotic and dead cells by Annexin V-FITC and PI and analyzed by flow cytometry (Fig. 1A). As determined in three independent experiments, the percentages of apoptotic/dead cells did not differ between mixed allogeneic DC cultures and each of the single donor-cultures. The percentage of living cells was actually more than $80 \%$ in the great majority of cases (Fig. 1B) and the difference between mixed donor and single donor cultures was negligible and therefore statistically insignificant (Fig. 2B).

\section{Long-term co-cultures of mixed allogeneic DCs display normal cell morphology}

In vitro morphology of monocyte-derived DCs depends on their maturation status, in addition to the quality of differentiation process, as well as subtle differences such as characteristic clustering upon activation with concomitant cell adhesion or formation of spindle-shape morphology, which are the consequence of different cell culture conditions. For this reason, we wanted to compare the basic DC morphology between single donor and mixed donor cultures. Using an inverted optical microscope we observed DC morphology after 48 hours of various cell culture con- 


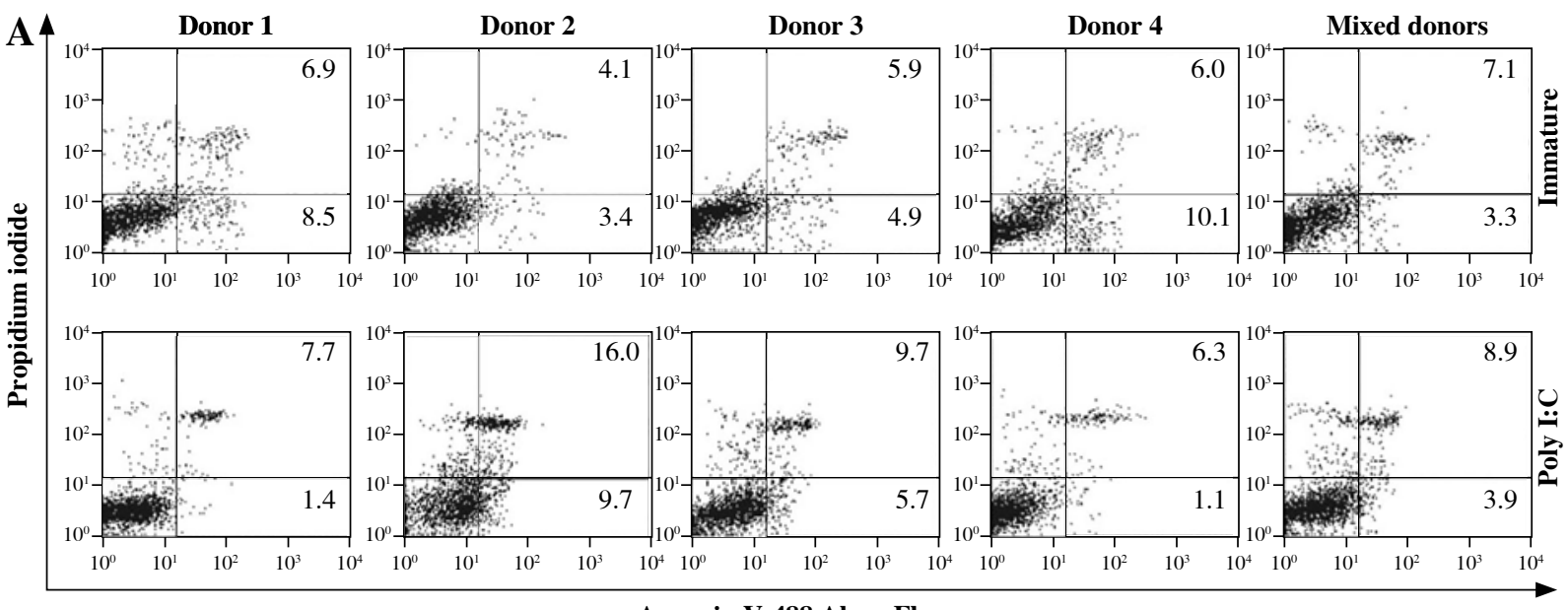

Annexin V-488 Alexa Fluor

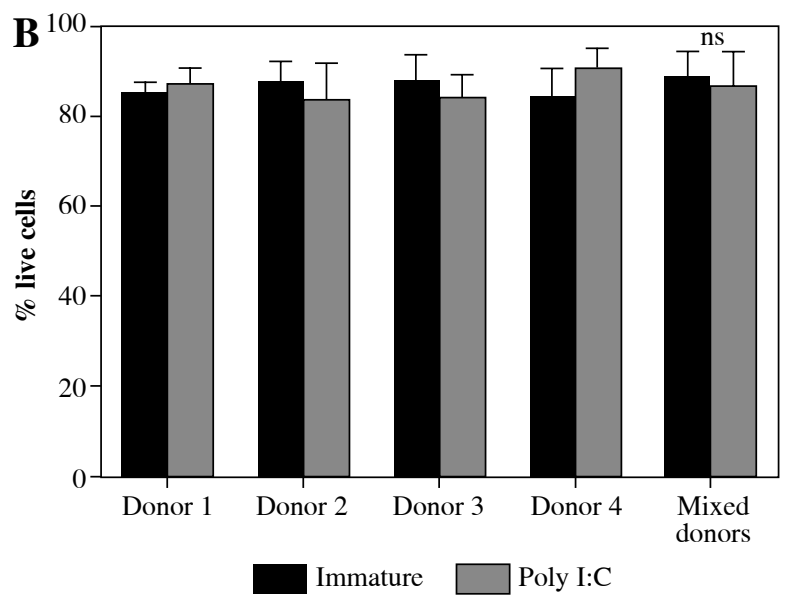

Fig. 1. Cultures of mixed allogeneic DCs are stable for longer periods in terms of cell survival. Dendritic cells were prepared and cultured as described in Material and methods. Differentiated from monocytes isolated from four unrelated healthy donors, the resulting iDCs were cultured both individually and in a co-culture, for 72 hours. Either single or mixed donor DCs were maintained in their immature state or matured with the addition of poly I:C (20 $\mu \mathrm{g} / \mathrm{ml})$. A) After 3 days the cells were stained with Annexin-FITC and PI to determine the percentages of apoptotic and dead cells. Numbers represent the percentages of single positive (apoptotic, lower right quadrant) and double positive (dead, upper right quadrant) cells. B) Percentages of living DCs in various cultures do not differ between mixed and single donor cultures. Significance between individual pairs was calculated using Student's unpaired $t$ test (ns - non-significant). The results shown in flow cytometry charts are representatives of 3 independent experiments

ditions, without additional activation stimuli, such as the addition of poly I:C or lipopolysaccharide (LPS) (except for the supplement of GM-CSF only). As can be seen in Figure 2, cell morphology in mixed allogeneic DC cultures displayed typical "potato"-shaped iDC morphology, with no excessive dendrite formation or cell clustering.

\section{DCs in mixed allogeneic cultures preserve stable immature phenotype and readily respond to maturation stimuli}

Next we wanted to determine the capacity of allogeneic DCs, kept in mixed co-cultures, to preserve their imma- ture activation state in the absence and their full maturation potential in the presence of exogenous maturation stimuli. For this reason DCs originating from monocytes of four unrelated donors were cultured for an additional 48 hours in single or mixed cultures without or with the addition of $20 \mu \mathrm{g} / \mathrm{ml}$ of the TLR3 agonist poly I:C. The cells were then stained for the expression of CD80 and CD86 co-stimulatory, chemokine receptor CCR7 and HLA-DR molecules. Figure 3 shows the expression levels of analyzed markers for both iDCs and mDCs. The characteristic iDC, CD80 ${ }^{\text {low }}$, $\mathrm{CD}^{\text {low }}{ }^{\text {lo }}$ CCR7 $7^{\text {low }}$, HLA-DR ${ }^{\text {med }}$ phenotypic profile was determined in all single donor, as well as in mixed donor DC cultures (Fig. 3A). In cultures that were treated with 


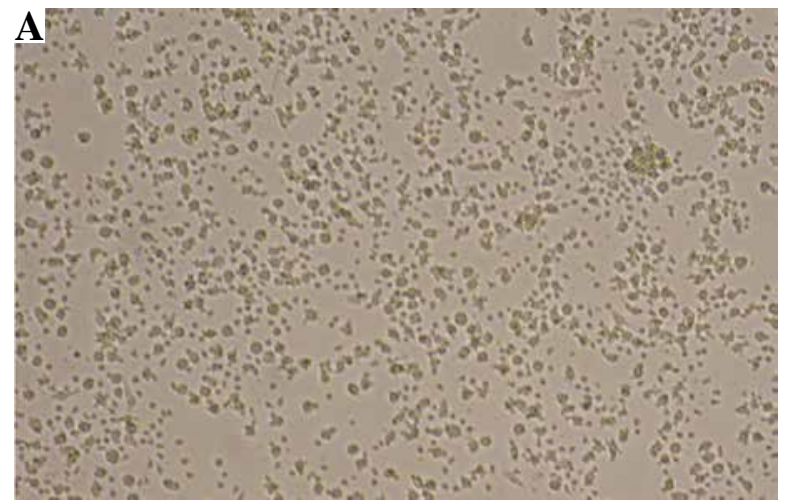

Single donor DCs

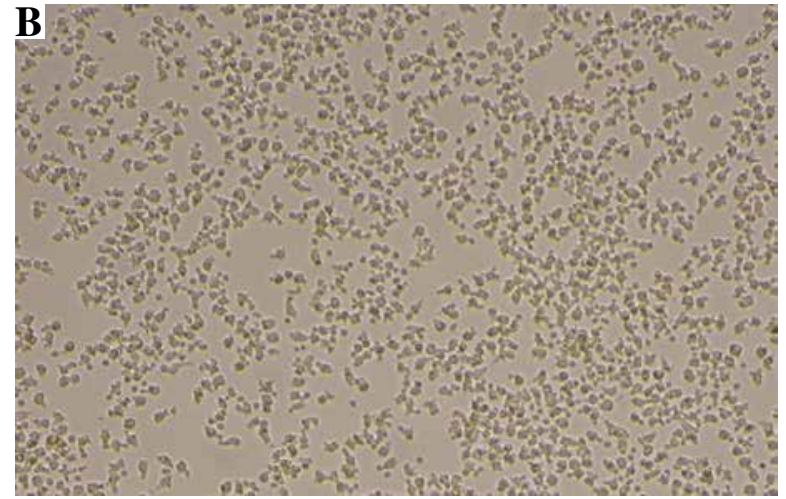

Mixed donors DCs

Fig. 2. Dendritic cells (DCs) from mixed allogeneic cultures display normal, immature morphology after prolonged cultivation time. DCs were differentiated from monocytes of four unrelated healthy donors. Fully differentiated iDCs were either cultured in single or mixed donor cultures for 72 hours. Cultures of mixed allogeneic DCs displayed no particular features in comparison to single donor ones. Normal immature morphology with the absence of cell clustering or adherence, which is characteristic of the DC maturation/activation process, was observed in both cases

$20 \mu \mathrm{g} / \mathrm{ml}$ of poly I:C, the iDCs matured readily, regardless of whether they contained cells from single or mixed donors, thereby extensively up-regulating all molecular markers, i.e. CD80, CD86, CCR7 and HLA-DR (Fig. 3B).

\section{Mixed allogeneic DCs maintain potent allo-stimulatory capacity}

To test for basic antigen-stimulating DC function, we set out to determine the capacity of variously cultured DCs to induce the proliferation of allogeneic $\mathrm{CD}^{+} \mathrm{T}$ cells. Immature DCs were first generated as described in Material and methods from monocytes of four unrelated donors and subsequently cultured in single and in mixed donor (all four donors combined) cultures. After 48 hours in the presence or absence of poly I:C maturation stimuli, the cells were used as allogeneic stimulators of responding $\mathrm{CD}^{+}$ $\mathrm{T}$ cells in a 5 days long mixed lymphocyte culture (MLC). On day 5 , the proliferation of alloactivated $\mathrm{T}$ cells was determined by liquid scintillation counting, for both iDCs and mDCs as stimulators (Fig. 4A, B). DCs from single donor and mixed donor cultures both exert strong capacity to stimulate the proliferation of allogeneic $\mathrm{CD}^{+} \mathrm{T}$ cells (Fig. 4B). As expected, in comparison to single donor DC stimulators, mixed donor DCs induce significantly more extensive proliferation of $\mathrm{T}$ cell responders, which stands for both iDCs and mDCs (Fig. 4A, B).

\section{DCs from mixed allogeneic cultures are able to produce IL-12p70 and induce potent Th1 responses}

Induction of potent Th1 effector responses is a prerequisite for effective anti-cancer DC vaccines. In the last step of our experimental work we evaluated the ability of variously cultured DCs to induce Th1 effectors from naïve $\mathrm{CD} 4{ }^{+} \mathrm{CD} 45 \mathrm{RA}^{+} \mathrm{T}$ cells during a 7-day co-culture. Since Th1 induction is dependent on the ability of DCs to produce IL-12p70, we first assessed the ability of DCs to produce IL-12p70 upon their maturation. As described in Material and methods, single or mixed donor DCs were activated with poly I:C for 48 hours. Subsequently, supernatants were sampled and analyzed for the presence of IL-12p70 and IL-10 (Fig. 5A). Dendritic cells from both single and mixed donor cultures were able to produce IL-12p70 upon maturation in quantities that have been reported to be characteristic for a single signal stimulation, i.e. with a defined toll-like receptor (TLR) ligand alone, without interferon (IFN)- $\gamma$ or CD40-ligand (average values of $774.3 \pm 116.4 \mathrm{pg} / \mathrm{ml}$ and $636.0 \pm 57.5 \mathrm{pg} / \mathrm{ml}$, for single and mixed DC cultures, respectively) [9].

In the next step we used these mDCs as in vitro stimulators of naïve $\mathrm{CD}^{+}{ }^{+} \mathrm{CD} 45 \mathrm{RA}^{+} \mathrm{T}$ cells. After 7 days in MLCs, responder T cells were collected, washed twice and stimulated with PMA and ionomycin, followed by the addition of brefeldin A. Cytokine production within the cells was determined with flow cytometry after intracellular staining for IFN- $\gamma$, IL-2, IL-4 and IL-10 (Fig. 5B, C). DCs in both single and mixed donor cultures induced effector T cell populations to produce low amounts of IL-4, IL-10 and high levels of IFN- $\gamma$ and IL-2. Where mixed donor mDCs were used as stimulators, the percentage of IFN- $\gamma$-producing T cell effectors increased for more than $50 \%$, compared to stimulating mDCs from single donor cultures $(21.7 \pm 5.9 \%$ and $38.7 \pm 10.5 \%$ of IFN- $\gamma$-producing $\mathrm{T}$ cells for single and mixed donor mDCs, respectively). 

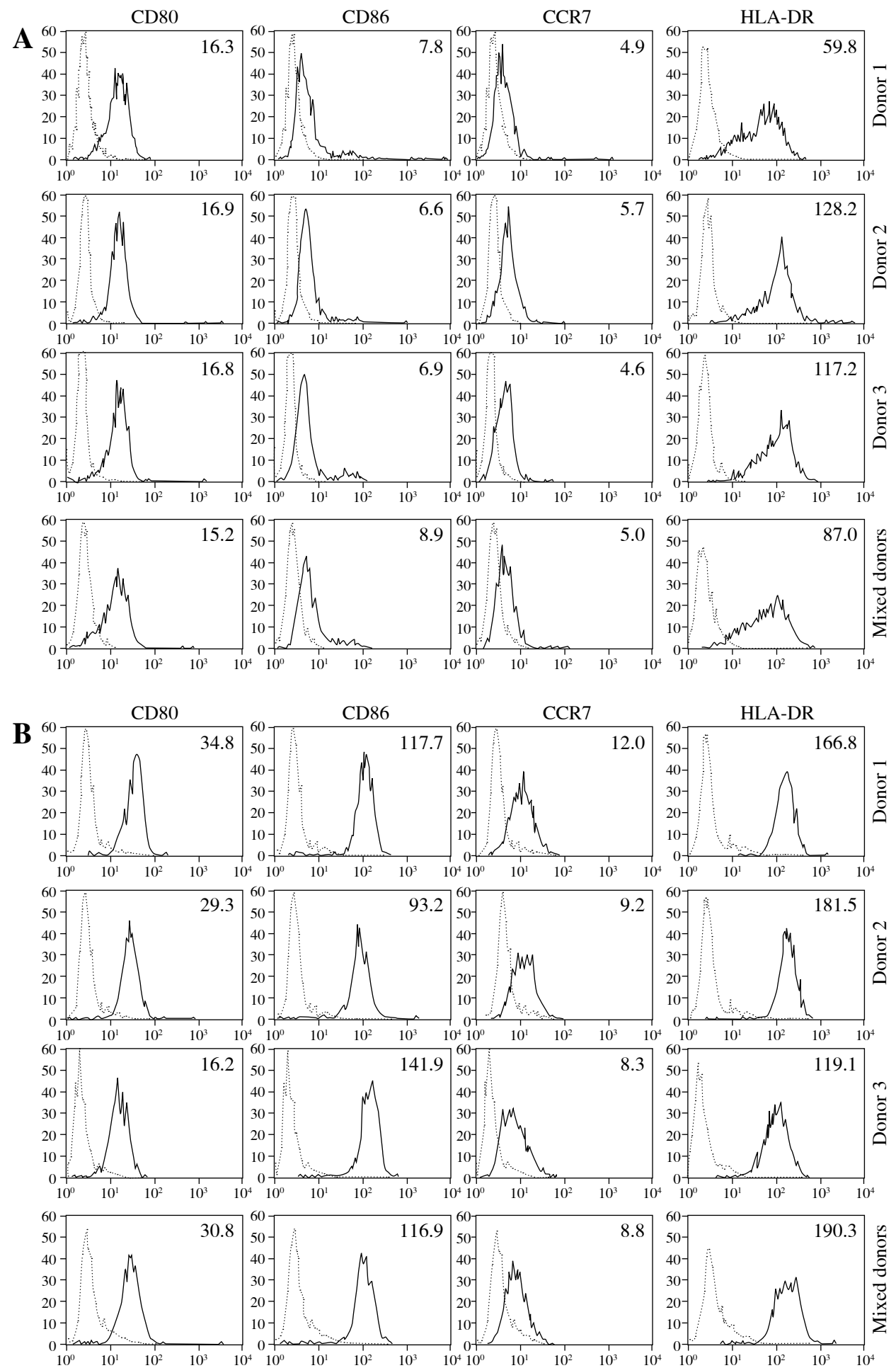

Fig. 3. Dendritic cells (DCs) from mixed allogeneic cultures preserve their immature phenotype and respond readily to maturation stimulus. DCs were prepared and cultured as described in Material and methods. Differentiated from monocytes, isolated from four unrelated healthy donors, the resulting iDCs were cultured both individually and in a co-culture, for 48 hours, either in the absence of presence of poly I:C $(20 \mu \mathrm{g} / \mathrm{ml})$. Subsequently, cells were harvested and stained for the depicted surface markers. Data show phenotypic characterizations of 3 out of 4 DCs from individual donors (only due to the clarity of the overview) and of DCs from a mixed culture. Numbers represent the mean fluorescence intensities (MFIs) for each depicted surface marker 

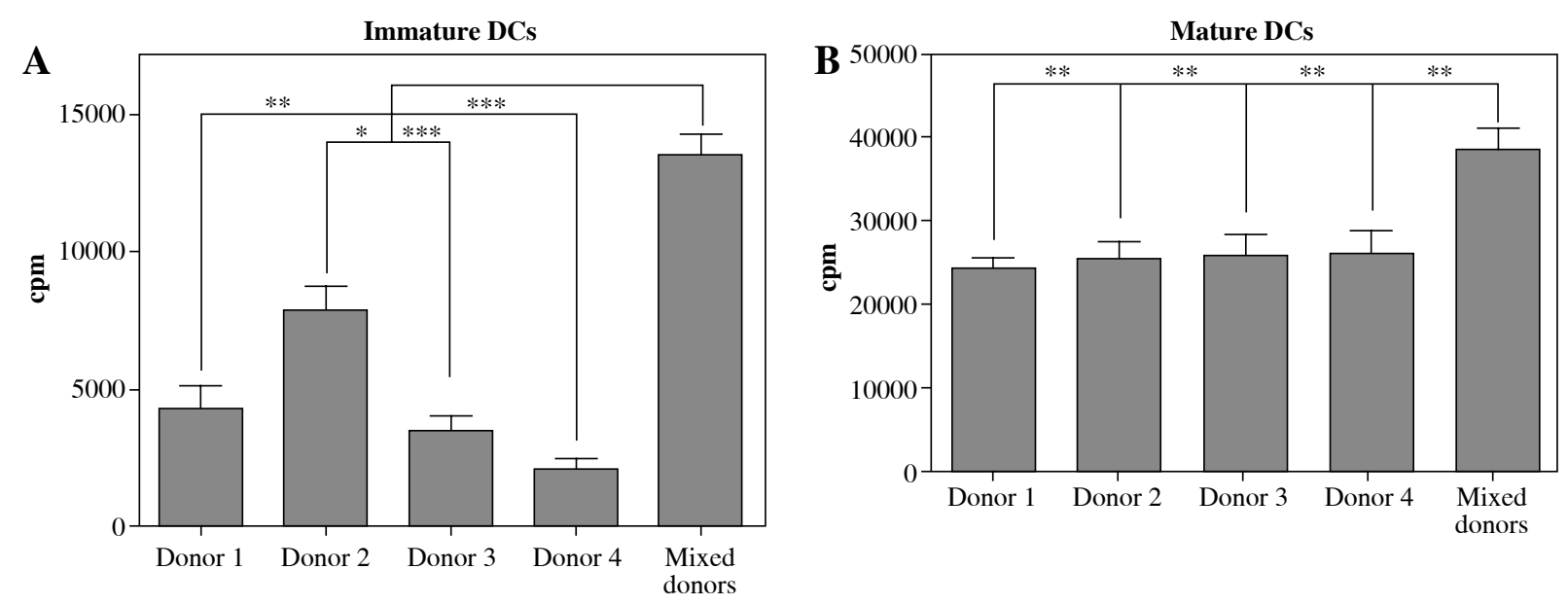

Fig. 4. Dendritic cells (DCs) from mixed allogeneic donor cultures possess potent allo-stimulatory capacity. The capacity of DCs to stimulate proliferation of naïve allogeneic $\mathrm{CD} 4^{+} \mathrm{T}$ cells was evaluated in a mixed lymphocyte culture (MLC). DCs were generated as described in Material and methods. DCs from single and mixed allogeneic donor cultures were used as stimulators either in their $(\mathbf{A})$ immature or $(\mathbf{B})$ mature (48 hour activation with $20 \mu \mathrm{g} / \mathrm{ml}$ poly I:C) state. Significance between compared pairs, as depicted in the figure, was calculated using Student's unpaired t test $\left(^{*} p<0.05\right.$, $* * p<0.01, * * * p<0.001)$. The results are presented as mean $\pm \mathrm{SD}$

\section{Discussion}

In the present study we show for the first time that after optimal differentiation, MoDCs from multiple unrelated donors can stably exist in in vitro co-culture and keep their unhindered functionalities. This demonstration is useful for cell-therapy purposes, but also intriguing from the scientific point of view. Namely, there have been demonstrations in the past that DCs do not act solely as couriers of antigenic information but also possess several mechanisms which grant them immune effector functions. Both myeloid and plasmacytoid DCs have been shown to exert direct cytotoxic effects, particularly against cancer cells [10]. This so-called DC "killer" effect has been demonstrated in both rodents and humans. In addition to circulating myeloid and plasmacytoid DCs, such effector functions have also been demonstrated for MoDCs. One of these direct cytotoxic mechanisms is associated with tumor necrosis factor (TNF)- $\alpha$-related apoptosis-inducing ligand (TRAIL). Intracellular TLRs such as TLR3 are capable of inducing TRAIL [11]. This was also demonstrated for type I IFNs, such as IFN- $\alpha$, which is also produced by MoDCs after their maturation induced by TLR 3 agonists, such as poly I:C. We have shown that mixed allogeneic DC cultures are stable in terms of cell survival even when fully differentiated iDCs were treated and matured with poly I:C (Fig. 1). In addition there are several potential cytotoxic mechanisms of DCs which include effector molecules, such as TNF- $\alpha[12,13]$, FasL [14, 15], lymphotoxin $\alpha$ and $\beta$ [12], caspase-8 [16], TNF-like weak inducer of apoptosis (TWEAK) [17] and IFN- $\gamma$ [18]. However, our results suggest that such mechanisms are most likely not exerted between MoDCs, even in an allogeneic setting under inflammatory conditions.

Direct recognition of DC-targeted cells, for example tumor cells, is still largely unknown. It has been shown that differentially activated plasmacytoid DCs (pDCs) can recognize and kill either MHC-I-positive (pDCs activated by intracellular TLR agonists, such as poly I:C or by IFN- $\alpha$ ) or MHC-I-negative cells (pDCs activated by Frühsommer-Meningoenzephalitis [FSME]) [19, 20]. To date there is no study confirming or disproving the ability of DCs to somehow recognize allogeneic MHC class I or II molecules on other allogeneic DCs. However, such a process would most likely proceed via direct cell-to-cell contacts. As can be seen from Figure 2, cultures of mixed allogeneic DCs did not display any additional increase of cell clustering even after prolonged cultivation time, suggesting allogeneic "invisibility" within this particular experimental context.

Since any potential allogeneic recognition would most likely result in a particular inflammatory response that would influence the activation state of DCs, we evaluated and compared the expression levels of molecular maturation markers on cells from mixed donor cultures and those from single donor cultures, following 3 days of cultivation. As can be seen in Figure 3A, DCs from mixed donor cultures possess low expression of CD80 and CD86 co-stimulatory molecules, lymph node-homing chemokine receptor CCR7 and HLA-DR molecules, which is very similar to that detected on single donor DCs. Therefore, in the absence of maturation stimuli, DCs in mixed allogeneic cultures remain immature also during longer periods of time. Activation of both types of DC cultures with poly I:C 


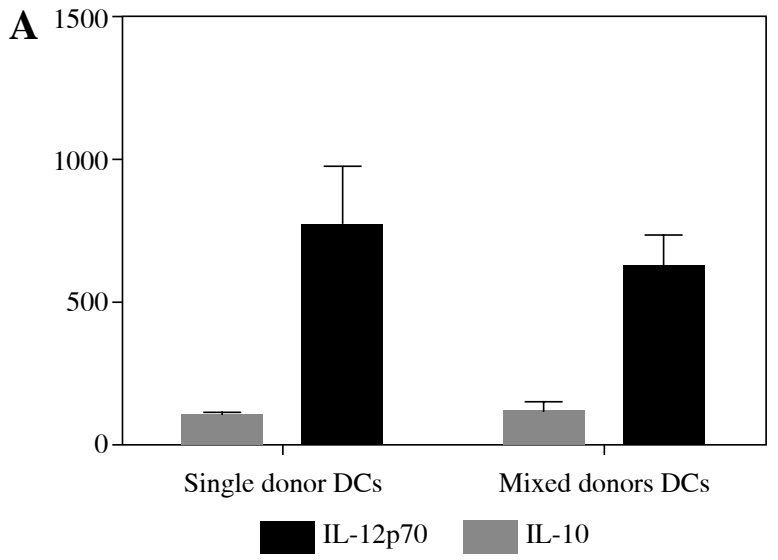

C

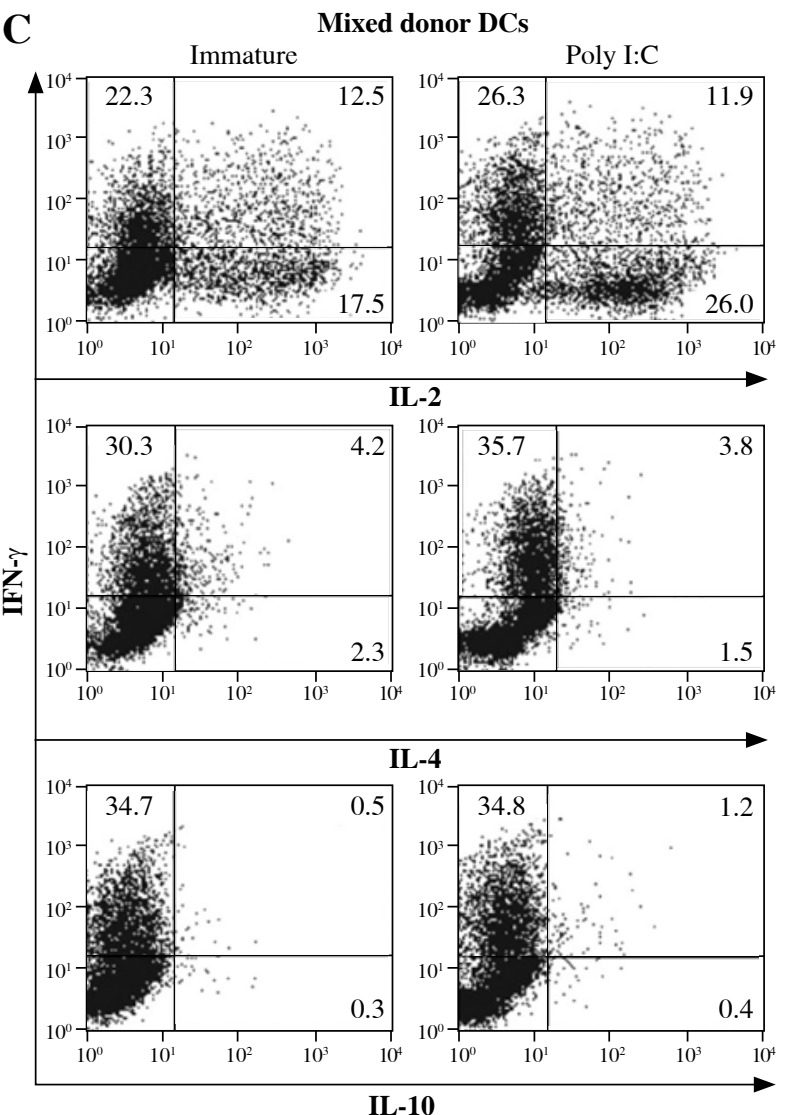

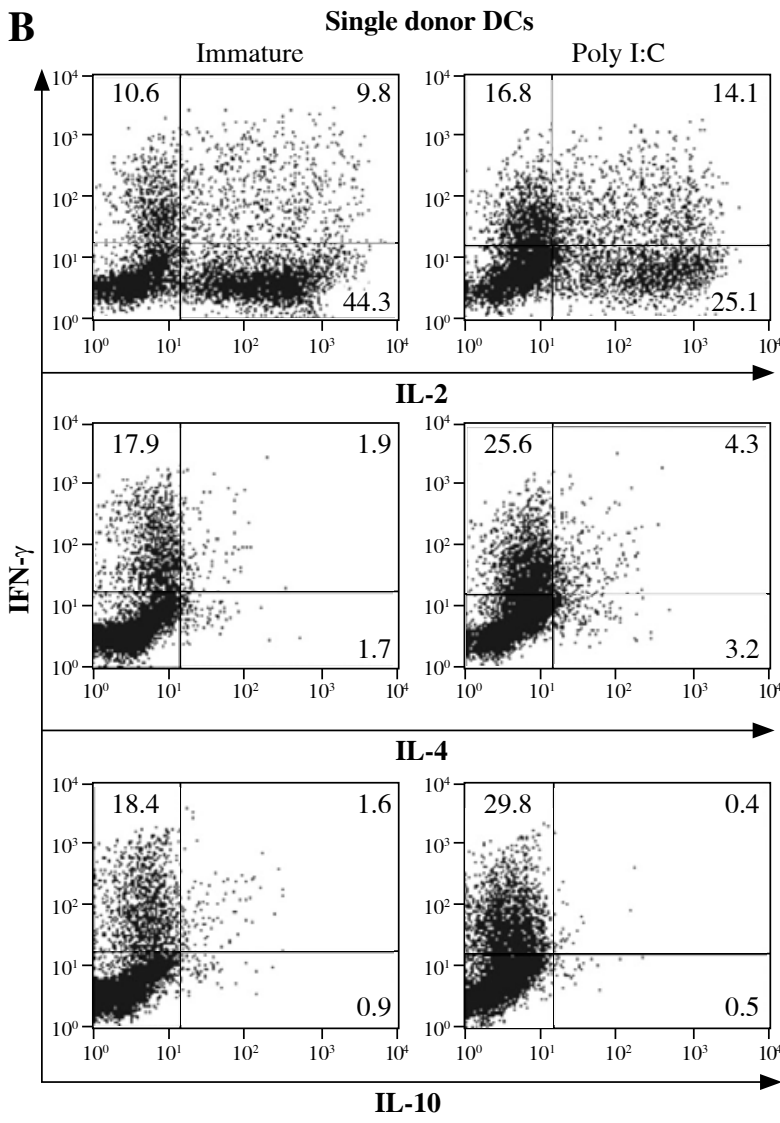

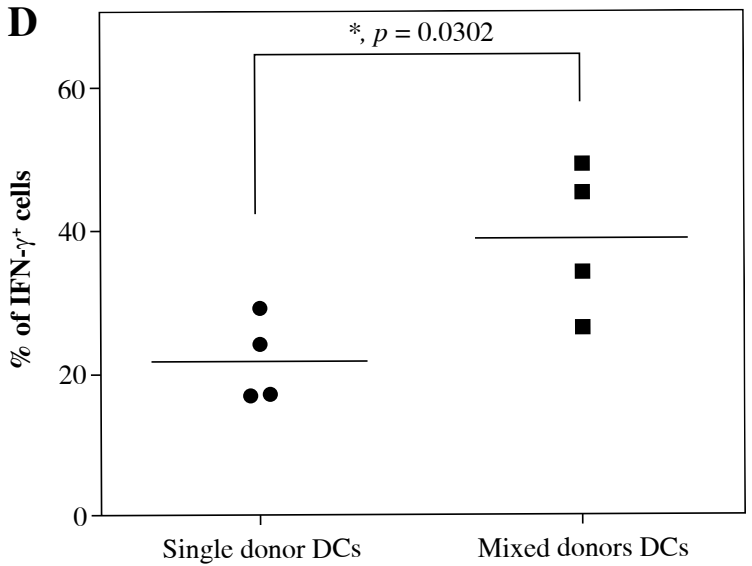

Fig. 5. Dendritic cells (DCs) from combined allogeneic donors induce potent Th1 effector responses. DCs were prepared as described in Material and methods and further cultured in either single or mixed donor cultures. A) After 48 hours, supernatants from both immature and mature (activated with $20 \mu \mathrm{g} / \mathrm{ml}$ poly I:C) DCs were taken and analyzed for the presence of IL-12p70 and IL10. B, C) Both immature and mature DCs were used as stimulators in 7 day MLCs with naïve $\mathrm{CD}^{+} \mathrm{CD} 45 \mathrm{RA}{ }^{+} \mathrm{T}$ cells as responders. After one week T cells were harvested, treated with PMA/ionomycin/ Brefeldin A and stained intracellularly for IFN- $\gamma$, IL-2, IL-4 and IL-10. The results were analyzed on a flow cytometer. One representative experiment out of four individual ones is shown. Numbers in quadrants represent percentages of cells stained for each particular cytokine. D) Statistical significance for percentage of IFN- $\gamma$-positive T cells is shown. The results where single or mixed allogeneic donor mDCs $(20 \mu \mathrm{g} / \mathrm{ml}$ poly I:C) were used as stimulators are presented. Statistical significance was calculated using Student's unpaired $t$-test $(* p<0.05)$ 
also resulted in successful maturation, confirmed by the $\mathrm{CD} 80^{\text {high }}, \mathrm{CD} 6^{\text {high }}, \mathrm{CCR}^{+}$, HLA-DR ${ }^{\text {high }}$ mature phenotype (Fig. 3B). Optimal DC maturation is a prerequisite for the preparation of quality anti-cancer DC vaccines, and their capacity to stimulate proliferation of tumor antigen specific responding $\mathrm{T}$ cell clones is basic for their functionality [21]. In the next step we evaluated the ability of differentially cultured DCs to induce proliferation of a third party, allogeneic $\mathrm{CD}^{+} \mathrm{T}$ cells (Fig. 4A, B). As expected, mDCs from all types of cultures were able to induce substantial proliferation of responding $\mathrm{T}$ cells, which was particularly extensive in the case of mixed allogeneic DCs. As we demonstrate, combined DCs from four different donors induced more than $50 \%$ greater $\mathrm{T}$ cell proliferation in comparison to single donor DCs. This effect was expected due to the greater HLA disparity and therefore increased percentage of allo-reactive T cells.

Although the exact mechanisms of anti-tumor effectiveness of DC vaccines are not completely resolved, there is now enough evidence to support the need for proper activation of anti-tumor specific cytotoxic $\mathrm{CD} 8^{+} \mathrm{T}$ cells, which requires a prior polarization of Th1 helper T cells [22]. Therefore we evaluated the capacity of DCs from single or mixed allogeneic donor cultures to induce Th1 polarization in a population of naïve third party $\mathrm{CD} 4^{+} \mathrm{T}$ cells (Fig. 5). Firstly, we determined the capacity of DCs to produce IL-12p70 in response to a single stimulus (poly I:C). Both mDCs in mixed and single donor cultures produced comparable levels of IL-12p70 (Fig. 5A). Afterwards, these $\mathrm{mDCs}$ were used as stimulators of naïve allogeneic $\mathrm{CD}^{+} \mathrm{T}$ cells in 7-day long MLCs. Dendritic cells from mixed donors generally induced $\mathrm{T}$ cell populations with an approximately $80 \%$ greater portion of IFN- $\gamma$-producing cells than single donor DCs (Fig. 5B-D). This capacity of mixed allogeneic donor DCs is attributable to greater HLA disparity in relation to responding $\mathrm{T}$ cells due to high diversity of the DC's HLA pool. Nevertheless, this clearly shows the capacity of mixed donor DCs to induce a strong Th1 responses and engage a greater number of responding $\mathrm{T}$ cell clones by expanded HLA recognition. While this effect might not influence the induction of selected peptide antigen-specific T cells, for example as in HLA-A2-restricted, peptide pulsed DC vaccines, it is speculated that allogeneic DC vaccines can support anti-tumor immunity in part through generation of pro-inflammatory conditions that support Th1-skewing and in part via direct priming of tumor-reactive or cross-reactive T cells $[23,24]$.

By strategically selecting multiple unrelated donors for mixed allogeneic DC vaccine manufacture, this could represent a platform for scalable vaccine manufacture that would include a range of HLA haplotypes applicable to a wide patient population. Interestingly, in a recent clinical study of a DCP-001 allogeneic DC vaccine, it was found that while HLA matches between vaccine and patients varied from 1 to 5 , the number of mismatches showed no clear correlation with patient survival [25]. The study showed that the use of DCP-001 vaccine in acute myeloid leukemia patients is safe, feasible and generates anti-tumor immune responses.

In conclusion, we have shown that the production of DC vaccines from primary human monocytes based on multiple allogeneic donors results in a functional cellular product and is a feasible approach towards development of future DC-based cellular products. Although the majority of anti-cancer DC vaccines are made from autologous sources, the use of allogeneic cellular vaccines might prove useful in manufacturing off-the-shelf DC vaccines and/or in cases where the physical state of cancer patient is unsuitable for the leukapheresis procedure.

\section{Acknowledgements}

The authors would like to thank Teja Germovnik, MPharm for her laboratory assistance. This study was supported by the research program P3-0371 of the Slovenian National Research Agency.

The authors declare no conflict of interests.

\section{References}

1. de Gruijl TD, van den Eertwegh AJ, Pinedo HM, Scheper RJ (2008): Whole-cell cancer vaccination: from autologous to allogeneic tumor- and dendritic cell-based vaccines. Cancer Immunol Immunother 57: 1569-1577.

2. Barbuto JA, Ensina LF, Neves AR, et al. (2004): Dendritic cell-tumor cell hybrid vaccination for metastatic cancer. Cancer Immunol Immunother 53: 1111-1118.

3. Hus I, Rolinski J, Tabarkiewicz J, et al. (2005): Allogeneic dendritic cells pulsed with tumor lysates or apoptotic bodies as immunotherapy for patients with early-stage B-cell chronic lymphocytic leukemia. Leukemia 19: 1621-1627.

4. Holtl L, Ramoner R, Zelle-Rieser C, et al. (2005): Allogeneic dendritic cell vaccination against metastatic renal cell carcinoma with or without cyclophosphamide. Cancer Immunol Immunother 54: 663-670.

5. Zhou J, Weng D, Zhou F, et al. (2009): Patient-derived renal cell carcinoma cells fused with allogeneic dendritic cells elicit anti-tumor activity: in vitro results and clinical responses. Cancer Immunol Immunother 58: 1587-1597.

6. Svajger U, Jeras M (2011): Optimal dendritic cell differentiation in rpmi media requires the absence of HEPES buffer. Immunol Invest 40: 413-426.

7. Padley DJ, Dietz AB, Gastineau DA, Vuk-Pavlovic S (2001): Mature myeloid dendritic cells for clinical use prepared from $\mathrm{CD} 14+$ cells isolated by immunomagnetic adsorption. $\mathrm{J}$ Hematother Stem Cell Res 10: 427-429.

8. Svajger U, Obermajer N, Jeras M (2014): IFN-gamma-rich environment programs dendritic cells toward silencing of cytotoxic immune responses. J Leukoc Biol 95: 33-46.

9. Vieira PL, de Jong EC, Wierenga EA, et al. (2000): Development of Th1-inducing capacity in myeloid dendritic cells requires environmental instruction. J Immunol 164: 4507-4512. 
10. Tel J, Anguille S, Waterborg CE, et al. (2014): Tumoricidal activity of human dendritic cells. Trends Immunol 35: 38-46.

11. Vidalain PO, Azocar O, Yagita H, et al. (2001): Cytotoxic activity of human dendritic cells is differentially regulated by double-stranded RNA and CD40 ligand. J Immunol 167: 3765-3772.

12. Lu G, Janjic BM, Janjic J, et al. (2002): Innate direct anticancer effector function of human immature dendritic cells. II. Role of TNF, lymphotoxin-alpha(1)beta(2), Fas ligand, and TNF-related apoptosis-inducing ligand. J Immunol 168: 18311839.

13. Joo HG, Fleming TP, Tanaka Y, et al. (2002): Human dendritic cells induce tumor-specific apoptosis by soluble factors. Int J Cancer 102: 20-28.

14. Yang R, Xu D, Zhang A, Gruber A (2001): Immature dendritic cells kill ovarian carcinoma cells by a FAS/FASL pathway, enabling them to sensitize tumor-specific CTLs. Int J Cancer 94: 407-413.

15. Zhao L, Tyrrell DL (2013): Myeloid dendritic cells can kill $\mathrm{T}$ cells during chronic hepatitis $\mathrm{C}$ virus infection. Viral Immunol 26: 25-39.

16. Vanderheyde N, Aksoy E, Amraoui Z, et al. (2001): Tumoricidal activity of monocyte-derived dendritic cells: evidence for a caspase-8-dependent, Fas-associated death domain-independent mechanism. J Immunol 167: 3565-3569.

17. Lichtner M, Maranon C, Vidalain PO, et al. (2004): HIV type 1infected dendritic cells induce apoptotic death in infected and uninfected primary CD4 T lymphocytes. AIDS Res Hum Retroviruses 20: 175-182.

18. Tomihara K, Kato K, Masuta Y, et al. (2008): Gene transfer of CD40-ligand to dendritic cells stimulates interferon-gamma production to induce growth arrest and apoptosis of tumor cells. Gene Ther 15: 203-213.

19. Tel J, Smits EL, Anguille S, et al. (2012): Human plasmacytoid dendritic cells are equipped with antigen-presenting and tumoricidal capacities. Blood 120: 3936-3944.

20. Kalb ML, Glaser A, Stary G, et al. (2012): TRAIL(+) human plasmacytoid dendritic cells kill tumor cells in vitro: mechanisms of imiquimod- and IFN-alpha-mediated antitumor reactivity. J Immunol 188: 1583-1591.

21. Hansen M, Met O, Svane IM, Andersen MH (2012): Cellular based cancer vaccines: type 1 polarization of dendritic cells. Curr Med Chem 19: 4239-4246.

22. Palucka K, Banchereau J (2013): Dendritic-cell-based therapeutic cancer vaccines. Immunity 39: 38-48.

23. Andersen BM, Ohlfest JR (2012): Increasing the efficacy of tumor cell vaccines by enhancing cross priming. Cancer Lett 325: $155-164$.

24. Joffre OP, Segura E, Savina A, Amigorena S (2012): Cross-presentation by dendritic cells. Nat Rev Immunol 12: 557-569.

25. van de Loosdrecht AA, van Wetering S, Santegoets $\mathrm{S}$, et al. (2018): A novel allogeneic off-the-shelf dendritic cell vaccine for post-remission treatment of elderly patients with acute myeloid leukemia. Cancer Immunol Immunother 67: 15051518. 